\title{
Activar archivos con narrativas sonoras
}

\author{
SÒNIA LÓPEZ ORTIZ \\ Curadora de contenidos digitales especializada en narrativas de audio para instituciones \\ culturales.
}

\begin{abstract}
En la actualidad asistimos, si no a una época dorada, sí sin duda a un momento de eclosión del formato llamado pódcast. Si hace unos años cualquiera que tuviera algo que contar lo hacía mediante un blog, hoy es el pódcast uno de los formatos mejor posicionados para generar notoriedad en torno a un tema. En este contexto, la narrativa sonora, a través del formato de los pódcasts, se presenta como una oportunidad para cumplir varios objetivos de cualquier institución con la misión de coleccionar, preservar y difundir. Por un lado, podemos añadir capas de interpretación a los archivos y colecciones; por el otro, diseminar esos contenidos en ecosistemas de nuevos públicos.
\end{abstract}

El propósito de este artículo es profundizar y analizar, a través de mi experiencia de más de 15 años de experimentación en diversos formatos, algunos casos de estudio y plantear algunas conclusiones acerca del potencial que el formato de narrativa sonora puede tener para los archivos y bibliotecas. La activación del archivo mediante la curaduría sonora proporciona un tejido que abriga el contenido, hablando directamente al oído del oyente, en un formato íntimo y seductor.

pódcast, audio, oralidad, narrativa, distribución, nuevos públicos, diseño sonoro

\section{Capturar procesos, plasmar lo intangible}

A lo largo de mi trayectoria profesional puedo decir que en muchas ocasiones he disfrutado del lujo de poder experimentar en mi día a día. Empecé a trabajar en medios digitales en 2001, cuando en el terreno de las instituciones e Internet todavía estaba todo por inventar. En esa fecha formaba parte del equipo de Programas públicos del Museu d'Art Contemporani de Barcelona (MACBA) y la coordinación de la página web era un proyecto más entre los varios que yo tenía asignados. Fue en 2004 cuando la web del Museo se convirtió en un proyecto a tiempo completo para mí, coincidiendo con mi traslado al departamento de Publicaciones del Museo. Este cambio proporcionó un contexto en extremo nutritivo para los proyectos digitales del MACBA. Por otro lado, me permitió abrir mi práctica profesional, formada en la historia del arte y entrenada en la coordinación y producción de actividades, a la sensibilidad de las publicaciones. También, me expuso a unos tiempos y procesos de trabajo totalmente diferentes a los que estaba habituada.

El tiempo de las publicaciones es seguramente el más largo de todos los que acompañan la tarea de investigación y gestación de una exposición. Si para los programas públicos de una

Bajo el principio de que el acceso abierto a los resultados de investigación acelera el avance del conocimiento, todos los contenidos de la edición electrónica de CLIP se distribuyen bajo una licencia de uso y distribución Creative Commons Reconocimiento-NoComercialCompartirlgual 3.0 España (CC BY-NC-SA 3.0 ES). 
muestra se trabaja con meses de antelación, las reuniones para la conceptualización de una publicación pueden empezar año y medio o dos antes de la inauguración. Esto, más que afectar a mi día a día de mantenimiento de contenidos de la web, sí tuvo impacto en mi forma de entender la gestación misma de los procesos de producción de la institución. Por el mismo despacho en el que yo tenía mi escritorio, veía pasar a comisarias, artistas, autores que hablaban, discutían e iban dando forma a proyectos futuros, mediante procesos intangibles. Ser testigo de estos procesos y entenderlos como parte fundamental de la misión pública de la institución, empezó a tener un impacto directo en mi forma de entender mi práctica profesional y la capacidad de experimentar y transformar los formatos con los que se hacían públicas líneas discursivas y proyectos del Museo. En este contexto la reflexión que me surgía era la siguiente: ¿cómo puedo contribuir a hacer de estos procesos, una publicación? ¿Qué medios, formatos, canales puedo abordar desde lo digital para ello? ¿Cómo puedo hacer de Internet una parte más de la esfera pública del museo, con una audiencia potencialmente global?

Lejos de querer aburrir al lector, me detengo en estos detalles que pueden parecer excesivamente circunstanciales, particulares y lejanos en el tiempo, porque marcaron una noción de publicación específica que, a mi modo de ver, sigue teniendo valor hoy en día. Los medios digitales y las oportunidades de diseminación que ofrecía Internet tenían que jugar un papel relevante como herramientas para la que la captura de todos esos procesos. Los nuevos medios digitales e Internet se presentaban como un espacio infinito para difundir y distribuir estos contenidos. Aunque hoy la web y la tecnología de las redes se han oscurecido ${ }^{1}$ en un giro aceleracionista y centralizador, en los 2000 todavía confiábamos en la utopía de la democratización del conocimiento que Internet auguraba.

Es en este contexto de cierta euforia positivista que oigo por primera vez hablar de pódcast. Me introduce en el término mi amigo y productor de radio Raül Hinojosa que me pone al día del debate abierto en el entorno de la radio generalista. Ante la creciente digitalización del medio y la disponibilidad de contenidos a la carta, se empezaba a cuestionar el impacto que estos factores tendrían sobre las audiencias y sus hábitos de consumo. Tras haber superado la amenaza del vídeo ¿iba el pódcast a matar a la estrella de la radio?²

Aunque desde un punto de vista estrictamente institucional los temores que este debate planteaba quedaban lejos de mis preocupaciones más inmediatas, sí supuso un contexto apropiado para ampliar los horizontes acerca de los formatos con los que podía trabajar. Alternativas para capturar procesos y comunicarlos fuera del texto o del paradigma visual, formas hegemónicas de la comunicación del Museo. ¿Por qué no experimentar con el registro oral y el formato radiofónico a la carta para hablar de otros aspectos de la institución? ¿Podía el formato del pódcast, aligerado de la infraestructura necesaria para la emisión de radio en directo, responder a esa necesidad?

\footnotetext{
${ }^{1}$ SEBASTIAN CHAN: Fire, Fire, Fire - words from Creative State 2019, En: Medium, https://sebchan.medium.com/fire-fire-fire-words-from-creative-state-2019-b314f33da1c4\#dbb0 Fecha de consulta: 7 de noviembre de 2021.

2 https://es.wikipedia.org/wiki/Video Killed the Radio Star
} 


\section{Ràdio Web MACBA}

Así nació en 2006 Ràdio Web MACBA (RWM), con pocos recursos, mucho entusiasmo y más intuiciones que certezas. Cómo apuntaba hace un momento, su gestación se basó en reflexiones como:

- ¿Qué herramientas ofrece Internet que nos permitan experimentar con la transmisión de las ideas y el conocimiento que se producen en nuestra institución?

- ¿Qué pasaría si intentáramos una forma diferente de acercar a nuestros visitantes de la web al contenido que el Museo produce constantemente?

- ¿Y si optáramos por un formato radiofónico accesible, familiar que permitiera a los usuarios acceder a este contenido cuando quisieran, bajo demanda?

Aunque empezó con una única línea de programación, en su origen Ràdio Web MACBA ya tenía visión de escalar hacia una parrilla de programación radiofónica más amplia. Creamos un dominio a parte porque queríamos significar que se trataba de un espacio más del Museo, con programación propia. El primer programa fue el Son[i]a, línea que a fecha de hoy sigue publicándose y que cuenta ya con 340 programas. En la etapa de lanzamiento publicamos dos o tres cápsulas cada semana de unos 10-12 minutos cada una. Con los recursos disponibles, este ritmo fue sostenible solo durante unos pocos meses. Pero las estadísticas pronto demostraron que había interés por parte de la audiencia, lo que nos animó a continuar. Además, el formato encajaba en nuestras expectativas y nos permitía crear nuevas redes de trabajo colaborativo, no solo con otras personas del equipo del museo, sino también con otros agentes de nuestro entorno que se sumaban a enriquecer el proyecto. Nos movíamos continuamente entre el amateurismo y la profesionalización.

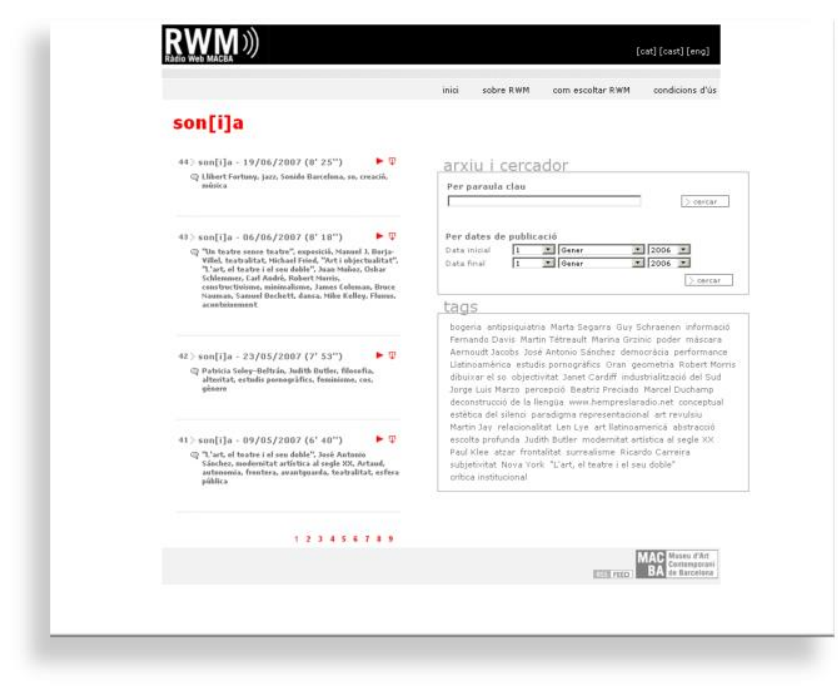

Figura 1: Ràdio Web MACBA en 2006. 
En 2008 Ràdio Web MACBA tuvo una primera ampliación, se incorporó una persona al equipo, mejoramos el diseño, la plataforma de gestión y también configuró la estructura que sigue conservando en buena medida hoy en día. Diversificamos la programación manteniendo el formato magazine del Son[i]a y se sumaron otras líneas de investigación como los programas Especiales que emergían directamente del trabajo con los comisarios y artistas que pasaban por el museo y desarrollamos líneas específicas más orientadas a la Investigación y los programas comisariados.

Hoy Ràdio Web MACBA es uno de los programas públicos más estables y de proyección internacional del Museo operando en la intersección entre el arte, las prácticas radiofónicas y la música experimental, con más de 800 archivos disponibles, casi 43.000 usuarios y 62.000 sesiones en $2020^{3}$.

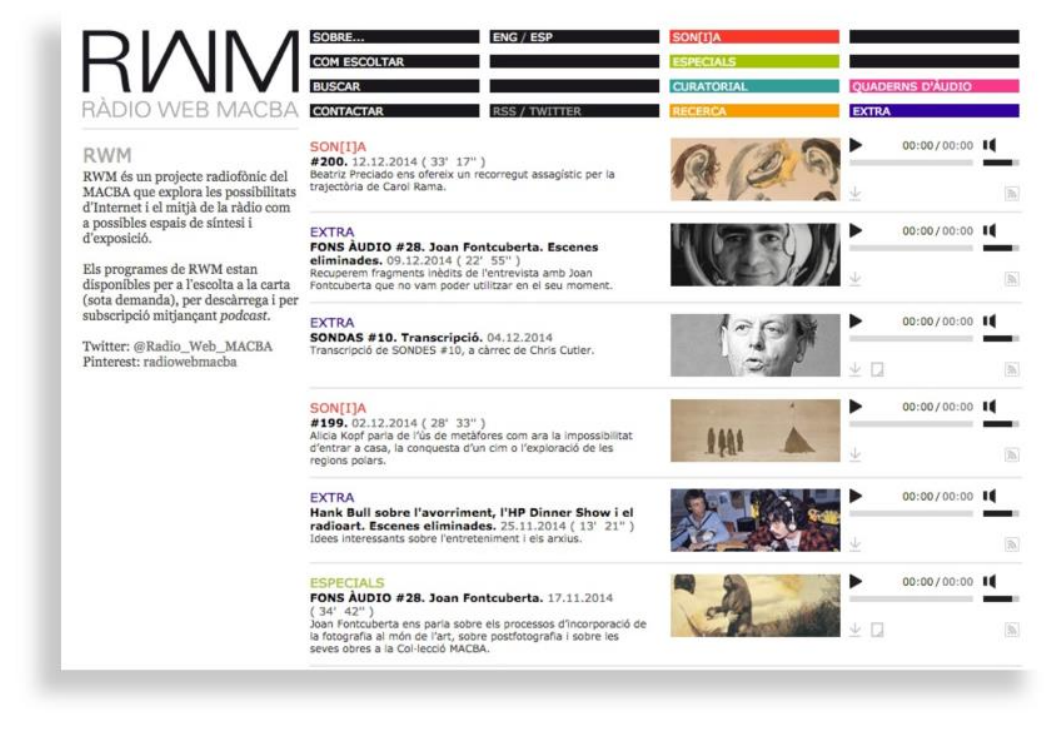

Figura 2: Ràdio Web MACBA en 2014.

\section{El pódcast como oportunidad}

Es tentador recrearse en la forma del proyecto de Ràdio Web MACBA ya que sin duda es relevante, porque en palabras de Marshall McLuhan: el medio es el mensaje 4 . Pero al fin y al cabo se trata de un proyecto que, aunque pionero, tiene ya más de 15 años. Pero el análisis detallado de la gestación del proyecto me sirve para aislar dos aspectos que aún hoy siguen pareciéndome esenciales en la práctica de la narrativa sonora y específicamente de la activación de colecciones y archivos mediante el registro de audio. Por un lado, las ricas posibilidades de la narrativa oral en un marco institucional eminentemente visual y textual; por el otro, la inclusión de la dimensión temporal, performativa (y por ende subjetiva) en la relación con la audiencia.

\footnotetext{
${ }^{3}$ Memoria MACBA 2020, pág 85. https://img.macba.cat/public/document/2021-09/memoria-macba-2020cast.pdf Fecha de consulta: 7 noviembre de 2021.

${ }^{4}$ MARSHALL MCLUHAN: Understanding Media: The Extensions of Man, 1964.
} 
En un momento en que el alcance de nuestros proyectos lo marcan en mayor medida, los algoritmos de los buscadores hegemónicos y las plataformas de las redes sociales, las instituciones tenemos que encontrar alternativas para recuperar el control de la calidad del tiempo de relación con nuestras audiencias. Si bien he perdido cierta confianza en otros formatos y medios, el pódcast sigue pareciéndome una oportunidad para que las instituciones nos posicionemos en nuestros propios términos oponiendo resistencia al frenesí efímero de otros formatos.

\section{Otros ejemplos}

Además de esta postura más bien teórica, las cifras también avalan este espacio de oportunidad. La Universidad de Navarra publica cada año la versión para España del prestigioso Digital News Report $^{5}$, iniciativa de la Universidad de Oxford y el Reuters Institute. Este informe estudia los hábitos de consumo y tendencias de los medios de comunicación digitales y es el estudio de referencia internacional sobre audiencias de pódcast. Según la edición más reciente, España es uno de los países de Europa Occidental donde más internautas escuchan pódcast con regularidad en el último mes: casi cuatro de cada diez (38\%). Además, se trata de una población joven, con intereses diversos y con frecuencia de naturaleza exploradora.

En cuanto a las preferencias de este público, lo interesante es que lo más escuchado no son los programas estrella de las radios generalistas (los deportivos y tertulias de gran audiencia en directo), sino de los espacios nicho, como los de divulgación histórica, que son un auténtico fenómeno.

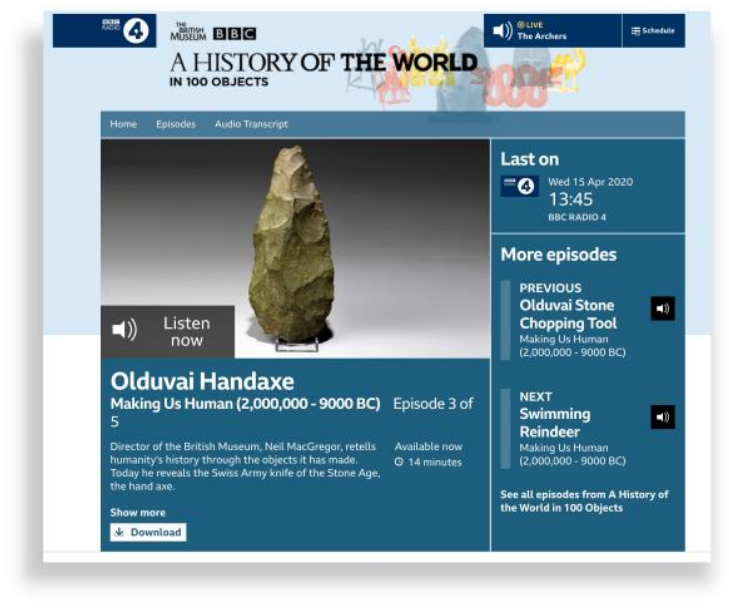

Figuraz: Selección del sitio web del pódcast A History of the World in 100 Objects.

\footnotetext{
5 https://www.digitalnewsreport.es/2021/el-consumo-de-pódcast-en-espana-permanece-estable/ Fecha consulta: 7 de noviembre de 2021.
} 
En este sentido, me gustaría destacar un proyecto de la BBC inglesa y el British Museum que fue pionero: el pódcast A History of the World in 100 Objects $^{6}$, en el que con una excelente factura sonora, Neil MacGregor, entonces director del museo, exploraba la historia de las civilizaciones a través de objetos de la colección permanente. Un proyecto que activaba todo el potencial para generar misterio, sorpresa, nuevas lecturas y el valor de piezas de la colección.

En España son excelentes ejemplos de creación de nuevas capas de interpretación a colecciones los pódcast de la Fundación Juan March. Por citar solo uno, en La biblioteca de Julio ${ }^{7}$ podemos recorrer los libros que acompañaron a Julio Cortázar durante su vida, y que se custodian en la Biblioteca de la Fundación. En cada capítulo se indaga en las anotaciones personales, los subrayados manuscritos de Cortázar, con el propósito de reunir a Cortázar con sus autores favoritos y, a todos ellos, con sus lectores-oyentes en el siglo XXI. Se trata pues de un proyecto que usa el medio del pódcast y su ecosistema de distribución para expandir la capacidad de alcance que tiene la propia biblioteca de la Fundación.

Fuera del ámbito institucional, quiero destacar un proyecto pequeño, particular y maravilloso que es un ejemplo singular de activación de una colección a través de la narrativa sonora. Se trata de Sent From Disneyland, un podcast semanal amateur que reconstruye la historia del parque temático más emblemático del mundo a través de postales de particulares enviadas desde Disneyland. Las postales, originalmente concebidas para el uso privado y el sentido de la vista — se miran y se leen-, adquieren una dimensión nueva al ser activadas por Clocky McDowell, el autor del pódcast. Se trata pues de una lectura con una óptica, visión y conocimiento que se sobreponen a las características originales del objeto. En Sent From Disneyland no solamente indagamos en la historia del parque, si no que especulamos también sobre las personas que escriben y reciben esas postales.

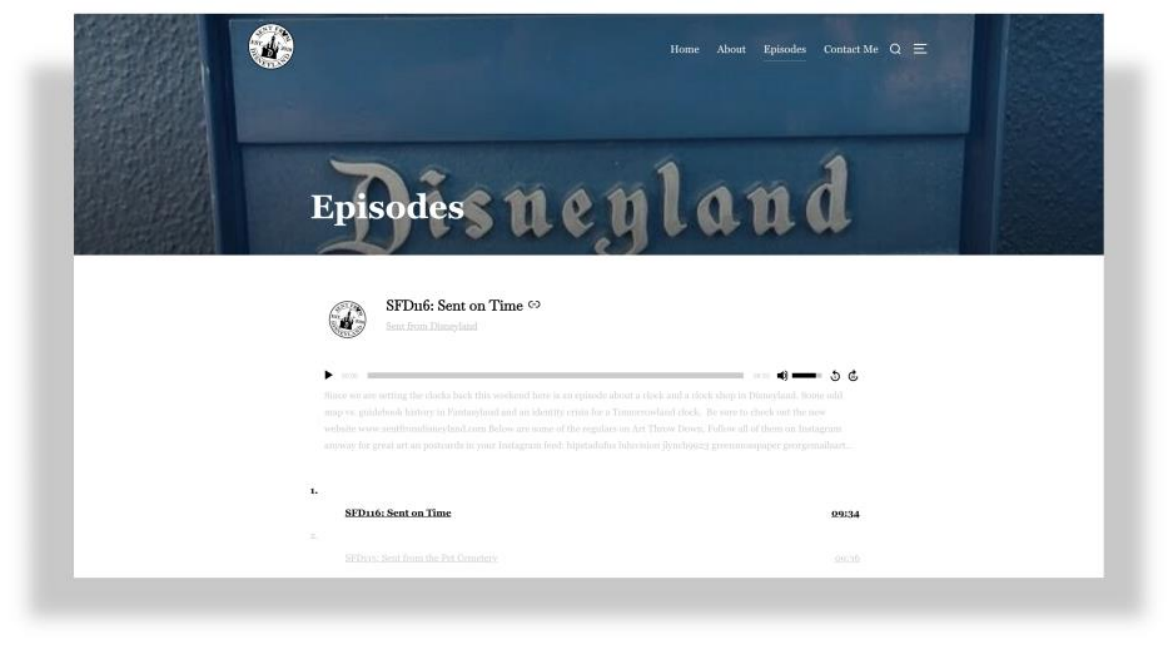

Figura4: Sitio web del pódcast Sent From Disneyland.

\footnotetext{
${ }^{6}$ https://www.bbc.co.uk/programmes/b00nrtd2/episodes/downloads? Fecha de consulta: 7 de noviembre de 2021.

7 https: / canal.march.es/es/podcast/biblioteca-julio?title $=\mathrm{Qu} \% \mathrm{C} 3 \% \mathrm{~A} 9+\mathrm{es}+\% 27 \mathrm{La}+$ biblioteca + de $+\mathrm{Julio} \% 27$
} Fecha de consulta: 7 de noviembre de 2021. 


\section{Conclusiones}

La narrativa sonora, el pódcast, ofrece, tanto a medios generalistas como a instituciones y amateurs, la posibilidad de recuperar lo que llamamos el formato del reportaje en profundidad, no necesariamente extenso en tiempo, si no literalmente profundo, específico, de nicho. Nos permite crear capas de contenido adicionales, prestar mayor atención a los detalles, dedicar más tiempo a temas minoritarios y aportar nuevas lecturas. Nos brinda la oportunidad de trabajar la subjectividad, la interpretación, darnos espacio para la especulación creativa.

En su conferencia Aura y temporalidad: la insistencia del archivo ${ }^{8}$, Wolfgang Ernst, profesor de Teoría de los Medios en el Instituto de Musicología y Estudios de Media de la Universidad Humboldt (Berlín) apuntaba que a los términos con los que se han definido los archivos tradicionalmente —orden espacial y clasificación-, hay que sumarles la temporalización. Esto permite la sincronización con el presente y ser relevante para el público de hoy. Ernst asigna al archivo el valor de ser mediador entre la memoria colectiva y el crecimiento personal del individuo y le otorga el atractivo de aparecer ante el público como el rincón de las instituciones que atesora los secretos ocultos.

Estas cualidades del archivo sumadas al poder de seducción que la narrativa oral y su capacidad de profundización especializada, se convierten en ingredientes de enorme atractivo para ensayar formas de activación de nuestros archivos y acervos de bibliotecas mediante series de pódcast.

\section{Referencias}

SEBASTIAN CHAN: Fire, Fire, Fire - words from Creative State, 2019. En: Medium. https://sebchan.medium.com/fire-fire-fire-words-from-creative-state-2019b314f33da1c4\#dbbo Fecha de consulta: 7 de noviembre de 2021.

LATITUDES: Itinerarios transversales: entrevista con Sònia López y Anna Ramos, 2012. https://www.Ittds.org/assets/OpenCurating_SoniaLopez-AnnaRamos.pdf. Fecha de consulta: 7 de noviembre de 2021.

WOLFANG ERNST: Aura and Temporality: The Insistence of the Archive, 2013. En: Quaderns Portàtils, MACBA (Barcelona). https://www.macba.cat/es/aprenderinvestigar/publicaciones/aura-and-temporality-insistence-archive Fecha de consulta: 7 de noviembre de 2021.

ANA ORMAECHEA Y PABLO FERNÁNDEZ DELKADER: Un viaje a través del audio, 2019. En: Revista Telos, Fundación Telefónica (Madrid). https://telos.fundaciontelefonica.com/telos-111-asuntos-de-comunicacion-ana-ormaecheapablo-fernandez-delkader-asi-escuchamos-un-viaje-a-traves-del-audio-consumo-podcast/ Fecha de consulta: 7 de noviembre de 2021.

\footnotetext{
8 https://www.macba.cat/es/aprender-investigar/publicaciones/aura-and-temporality-insistence-archive Fecha de consulta: 7 de noviembre de 2021.
} 


\section{Sobre la autora:}

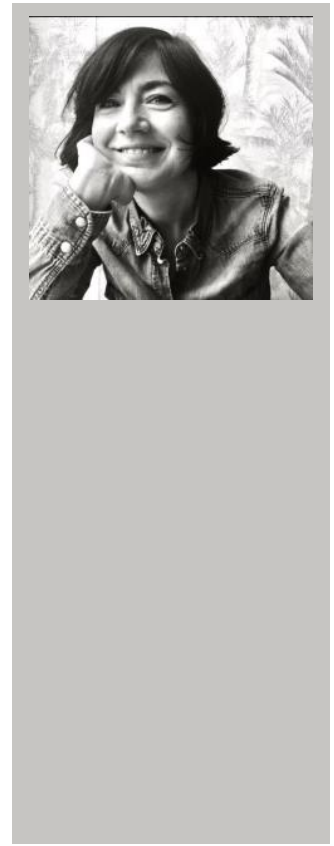

\section{SÒNIA LÓPEZ ORTIZ}

Curadora de contenidos digitales para instituciones culturales. Fundadora del sello de pódcasts Deckard.

Licenciada en Historia del Arte por la UB. Experta en curadoría digital, formatos de distribución de contenidos, arte, cultura, divulgación y comunicación. Responsable de Medios Digitales del MACBA de 2001 a 2017, fue la fundadora y directora de la premiada Ràdio Web MACBA, pionera en el uso del formato pódcast y la experimentación radiofónica en el contexto museístico. Entre los proyectos más recientes se cuentan la producción ejecutiva del proyecto Archivo Oral Picasso, iniciativa del Museo Picasso Barcelona, el Museo Reina Sofía y el Museo Picasso de París. Recientemente ha realizado proyectos sonoros para la Agència Catalana de Patrimoni, el Institut Ramon Llull y el MeV Museu d'Art Medieval.

- Web: https://sonialopez.es/

- Web: https://deckardproject.com/

- Email: lopesita@gmail.com

- Instagram y Twitter: @lopesita 